\title{
Quantum Communication Through Spin Chain Dynamics: An Introductory Overview
}

\author{
Sougato Bose \\ Department of Physics and Astronomy, University College London, Gower St., London WC1E 6BT, UK
}

(Dated: October 23, 2018)

\begin{abstract}
We present an introductory overview of the use of spin chains as quantum wires, which has recently developed into a topic of lively interest. The principal motivation is in connecting quantum registers without resorting to optics. A spin chain is a permanently coupled 1D system of spins. When one places a quantum state on one end of it, the state will be dynamically transmitted to the other end with some efficiency if the spins are coupled by an exchange interaction. No external modulations or measurements on the body of the chain, except perhaps at the very ends, is required for this purpose. For the simplest (uniformly coupled) chain and the simplest encoding (single qubit encoding), however, dispersion reduces the quality of transfer. We present a variety of alternatives proposed by various groups to achieve perfect quantum state transfer through spin chains. We conclude with a brief discussion of the various directions in which the topic is developing.
\end{abstract}

\section{INTRODUCTION}

Quantum communication is the act of transferring a quantum state from one place to another. By far its most well known application is quantum key distribution through which a secret random key can be established between distant parties with its security guaranteed by quantum mechanics [1, 2]. In quantum key distribution, a quantum state prepared by one party only needs to be measured by another party at a distance. For this purpose, photons are very well suited as they easily travel long distances through optical fibres or empty space and can be readily measured by a receiving party. Increasingly, however, the pivotal importance of quantum communication in a different area of quantum information processing, namely quantum computation, is becoming clear. It is becoming important for connecting up distinct quantum processors or registers to make a powerful quantum computer [3, 4, 5]. For this application, it is not only important to transfer a quantum state between locations but also to map it from/to the elements of the quantum register sending/receiving it. This necessitates simple exchange of quantum information between the elements of a quantum computer and the entities carrying the information between the computers. Moreover the transfer is needed only over short distances separating distinct registers. For such short distance applications, where mapping of the transferred quantum state to the elements of a register is also important, it is very useful to have alternatives to photons [3, 5]. This review article will be based on one such alternative, where the quantum state transfer is accomplished purely through the natural dynamical evolution of a permanently coupled chain of quantum systems, which has recently drawn considerable attention $6,7,8,9,10,11,12,13,14,15,16,17,18,19$, 20, 21, 22, 23, 24, 25, 26, 27, 28, 29, 30, 31, 32, 33, 34, 35, 36, 37, 38, 39, 40, 41, 42, 43, 44, 45, 46, 47, 48, 49, 50, 51, 52, 53, 54, 55, 56, 57, 58, 59, 60, 61, 62, 63]. We start by highlighting the importance of short distance quantum communications in quantum computation and why nonphotonic alternatives are important to pursue. At this point it is worth mentioning that dynamics is certainly not the only way to transfer a quantum state through a chain of permanently coupled quantum systems. Stationary states (such as thermal and ground states) of manybody systems have been studied from the quantum information theoretic viewpoint for quite a while (see, for example, Refs. 64, 65, 66, 67, 68]) and recently it was realized that certain classes of such chains accomplish the perfect transfer of a quantum state through measurements [69], while certain other classes can accomplish the same even without measurements [70, 71]. However, in this review, I will concentrate only on the literature on the use of spin chain dynamics for quantum communications.

\section{A. Quantum Communication for Connecting Quantum Registers/Computers:}

Quantum Computers, when realized, hold the promise of speeding up the solution of certain problems perceived as difficult on a classical computer [72, 73, 74, 75]. They also hold the promise of enabling controlled simulations of the behaviour of complex quantum systems [75, 76]. The typical quantum computer is regarded as a collection of quantum two state systems (or "qubits") on which arbitrary unitary operations can be performed, as for example shown in Fig 1, The power of a quantum computer increases with the number of qubits. However, there are several fundamental obstacles to increasing the number of qubits in a quantum computer arbitrarily. For example, the computer may be based on a common bus through which the qubits interact [77, 78, 79], as depicted in Fig.1. Then there is a physical limitation on the number of qubits which can be linked by the same bus. Alternatively, if the computer is based on direct interactions between qubits [80, 81, 82], as also shown in Fig,1, then either the qubits have to be moved close enough to interact, or the states they bear have to be transferred to qubits which are already within the range of each other's interaction. Thus the size of an individual quantum computer will be limited by the need to maximize efficiency. One way to get around the above problems is to envisage 


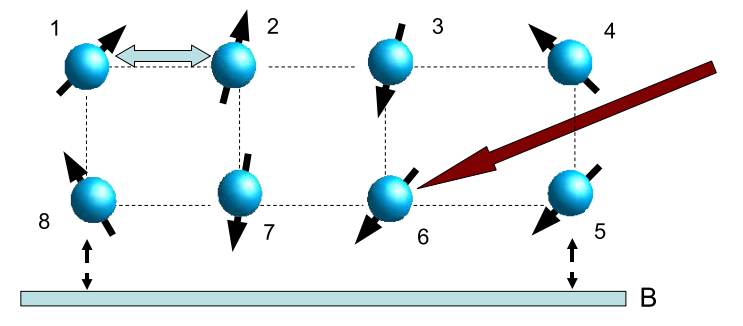

FIG. 1: The figure shows a typical quantum computer. The spheres with arrows denote qubits such as spin- $1 / 2$ systems. Typically operations on individual qubits are required, and accomplished by local fields on individual qubits, as shown, for example, by the long arrow controlling qubit 6 . Moreover, any pair of qubits are required to interact with each other. This can take place through a common bus. For example, qubits 5 and 8 are shown to be interacting through the common bus B. Naturally, the number of qubits which can be accommodated on a single bus is limited, and this restricts the size of feasible computers. An alternate strategy, in computers without a common bus mode is to make qubits interact directly. In those cases, if one intends to make joint operations between distant qubits, then their states have to be transferred to neighbouring qubits, such as qubits 1 and 2 , and then these qubits will be made to interact, as shown. For efficient functioning of the computer, the time-scale of the transfer must be restricted, which again limits the size of the computer.

a quantum computer composed of a number of quantum registers connected to each other by quantum communication channels (which could effectively be physical shuttling of qubits [3, 5]). Most operations would take place between qubits of the same register through, say, a common bus mode, or direct interactions together with very fast qubit transfers within the register. Occasionally, the quantum channels would be used to transfer qubits from one register to another, and thereby enable quantum gates between qubits of different registers. In contrast to the quantum channels required for quantum key distribution, these channels inside a quantum computer could be very short and should allow significant pre and post processing of the quantum state communicated through the channel in the transmitting and receiving registers.

Even if we are able to scale up quantum computers by some technology which does not require internal communication channels, such channels will still be necessary to hook up distinct quantum computers. For reasons of

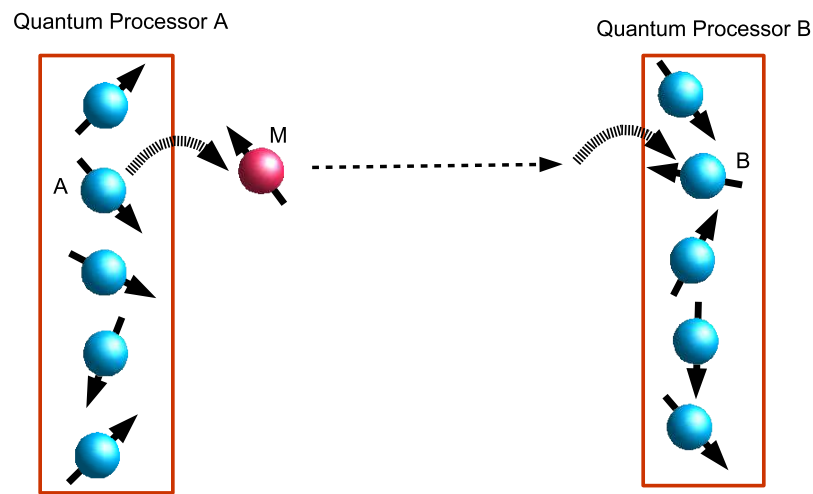

FIG. 2: The usual approach to connecting quantum processors. Quantum information stored in the stationary (blue) qubits of one register is mapped to a mobile (red) qubit. This qubit flies to the other register, where the information stored in it is mapped on to the stationary (blue) qubits of that register.

compactness, mobility and cost, we might just prefer to have small sized quantum computers. However at times one may need to tackle very complex problems for which the power of a single quantum computer will not suffice. It will then become very important to combine the processing powers of distinct quantum computers to obtain a computer with a greater processing power. Again, in contrast to the photonic channels currently being used for quantum key distribution, these need not be long (the computers could be sitting next to each other). Moreover, as the channel connects quantum computers, some encoding and decoding of the state should easily be possible inside the quantum computers. The transmitted state (after decoding, if applicable) should be transferable to a qubit or a group of qubits of the quantum computer that receives it.

\section{B. An unmodulated chain of quantum systems as a communication channel between quantum computers}

The usual approach envisaged for connecting quantum computers (or processors) is to first map the state to be transmitted from the qubits of one processor to a flying or mobile qubit. This flying qubit then traverses through a channel and reaches a second processor, where its state is mapped on to the qubits of that processor. The usual approach is depicted in Fig,2. However, depending on the physical nature of the qubits of the processors, this approach could involve either (a) interfacing between different physical systems such as stationary spins and photons 83] or stationary and mobile spins [84] or (b) physically 
moving a quantum system and subsequently bringing it to a halt elsewhere such as shuttling ions [3] or electrons [5]. All the above can be complicated in many respects. So one can ask the question: is it possible to transfer quantum information from place to place using only stationary qubits? The first idea that comes to mind is to have a chain of qubits as shown in Fig 3, and swap a quantum state perfectly in succession from one qubit to the next. A quantum state encoded on a qubit at one end of the line can be transported perfectly through a series of swaps to the qubit at the opposite end of the line. For example, in Fig 3, the strategy is to swap state in the following order: $A \rightarrow 1,1 \rightarrow 2,2 \rightarrow 3,3 \rightarrow 4,4 \rightarrow 5,5 \rightarrow B$. This kind of data-bus, called a swapping channel, has been discussed, for example, in Ref. 85]. However, such a data-bus requires the ability to modulate the strength or nature of interactions between pairs of adjacent qubits (such as 1 and 2 or 3 and 4 ) in time. Typically, this would require control fields on the wire varying over the scale of the spacing between the qubits. If so much control is available on a chain of qubits, then why not use the chain as a quantum computer? It will then be a gross underutilization to use it merely as a data-bus. Moreover, the requirement of so much control for the transfer of a quantum state naturally implies that such a protocol is also very susceptible to errors in these controls. For example, there are 6 pair-wise interactions to be switched on and off in succession for transmission of the state of qubit $A$ to that of qubit $B$ in Fig,2, and errors would accumulate in each of these steps. Thus the question arises as to whether we can utilize systems with much lower controls for connecting quantum registers. For example, if the interactions between qubits in a chain are permanent and uncontrollable (always on and constant in strength), and we are not allowed to apply any control fields to the qubits, could the chain still act as a quantum data-bus? The validity of the above possibility will enable us to use such a qubit chain as a data-bus in the true sense of word. This is because in the normal everyday use of the word "data-bus", such as to denote a cable connecting two computers, we do not envisage controlling every individual part of the cable and we mostly let the information flow through it in its own natural way. A qubit chain in which inter-qubit interactions are permanent, is an example of a spin chain, which we introduce below.

\section{Spin chains and the exchange interaction}

As the reader will know, in quantum mechanics, spins are systems endowed with tiny quantized magnetic moments. Bulk materials often have a large collection of spins permanently coupled to each other. The mutual interactions of a of these spins makes them prefer alignment or anti-alignment with respect to each other, resulting in diverse phenomena such as ferromagnetism and anti-ferromagnetism. A spin chain models a large class of such materials in which the spins are arranged in a one di-

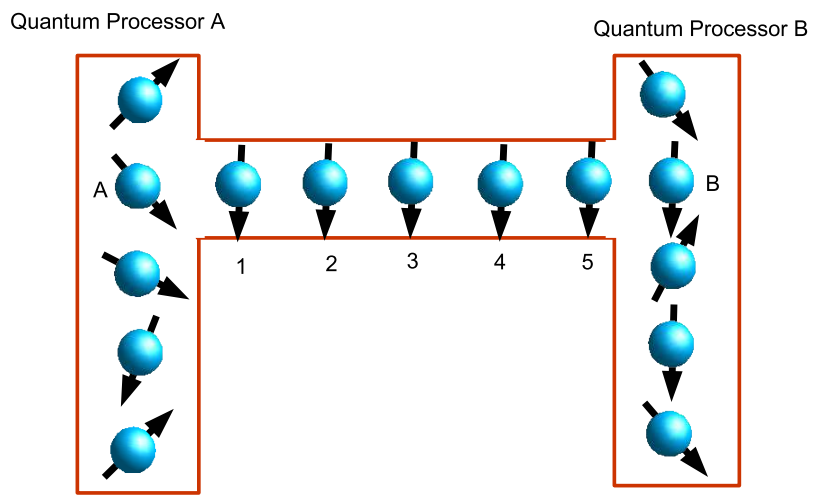

FIG. 3: This figure depicts the possibility of transporting quantum information from one quantum processor to another through a line of stationary qubits.

mensional lattice and permanently coupled to each other, usually with an interaction strength decreasing with distance (as shown in Fig 4). A common form of the Hamiltonian for the interaction between the $i$ th and the $j$ th spin is written as

$$
\mathbf{H}_{i j}=J_{i j} \vec{S}_{i} \cdot \vec{S}_{j},
$$

where $\vec{S}_{i} \cdot \vec{S}_{j} \equiv S_{i}^{x} S_{j}^{x}+S_{i}^{y} S_{j}^{y}+S_{i}^{z} S_{j}^{z}$ and $S_{i}^{x}, S_{i}^{y}, S_{i}^{z}$ are the operators for the component of the $i$ th spin along the $x, y$ and $z$ directions respectively. In particular, when all the spins are spin-1/2 systems, $S^{x}, S^{y}$ and $S^{z}$ stand for the familiar Pauli matrices $\sigma^{x}, \sigma^{y}$ and $\sigma^{z}$. A Hamiltonian of the above form is termed as an exchange interaction as it can arise in from the pure exchange electrons between neighbouring ions in a metal. It is also called the Heisenberg interaction after its inventor. In particular, the specific Hamiltonian we have written above is called the isotropic exchange interaction. In this paper we will also encounter a variant of the above interaction

$$
\mathbf{H}_{i j}^{X Y}=J_{i j}\left(S_{i}^{x} S_{j}^{x}+S_{i}^{y} S_{j}^{y}\right)
$$

which is called the XY interaction. We will be primarily concerned with chains of spin- $1 / 2$ systems in this article. Not only do examples of such systems exist in nature [86], but also can be fabricated in systems of any kind of qubits [25, 42, 51], as qubits are isomorphic to spin- $1 / 2$ systems. If one indeed fabricates artificial systems, why would one fabricate a spin chain i.e., a system with permanent couplings rather than a system where such couplings are also switchable? The obvious answer is that such a system should have a much lower complexity of fabrication because they do not require the an attached mechanism (such as electrodes) varying over the scale of the separation of the qubits to modulate their interactions. 


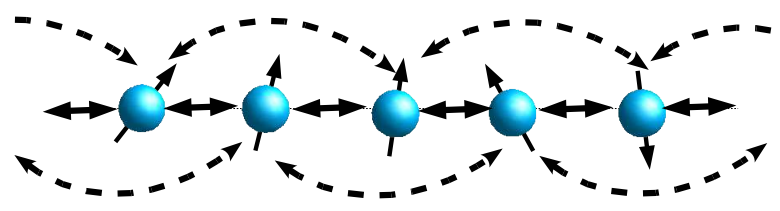

FIG. 4: The figure shows a spin chain: a system of spins perpetually coupled to each other with an interaction strength which generally decreases with distance. The double arrowed lines depict interactions with the dotted line denoting a weaker strength than the solid line.

\section{Quality check for a qubit array}

We now provide a couple of other motivations for studying quantum communications through a spin chain, quite distinct to the simple aim of connecting quantum registers. Suppose a linear array of qubits has been fabricated to function as a quantum computer. One then needs to check whether each qubit behaves as a bonafide qubit: namely they are able to remain in a quantum superposition of two distinct states for a time longer than that needed for running an entire quantum algorithm in the computer. Such superpositions may be destroyed by the interaction of a qubit with its environment, a process known as decoherence. The most straightforward strategy will be to probe each qubit individually by first switching off a qubit's interactions with adjacent qubits, initializing it in a known state and then applying a set of known unitary operations to it and measuring its state at the end. This strategy is very time consuming for a long array. An easier way is just to test the ability of the array to behave as quantum communication channel. One has to place a known quantum state on the qubit at one end of an array, switch on the interactions of each qubit with its immediate neighbours (so that the qubit array is now isomorphic to a spin chain with nearest neighbour interactions) and probe how well the state is retrieved from the other end after a specified interval of time. This requires initialization and measurements on only the two qubits at the ends of the array. As the quantum state has to pass through all the intermediate qubits in order to be transmitted from one end of a linear array to another, the ability of each qubit to behave as a bonafide qubit is automatically tested. If there are a few faulty qubits in the array (faulty means that they decohere significantly in the time-scale of the experiment), then the quality of the state transmission will be much lower than that expected for a fault-less array. Thus quantum communication through an array of qubits can enable a quality test of the entire array by just probing two of the qubits.

\section{E. Quantum response to a quantum impulse}

Typically, one characterizes the behaviour of complex systems by how it responds to external stimuli. Usually a classical field is applied to some part of the system and the resulting change in the value of some variable at a different part of the system is determined. For example, one can characterize magnetic systems by their magnetic susceptibility, which can determine how a magnetic field applied to one part of the system affects the magnetization in a different part of the system. Knowledge of this response enables the design of components which may use the magnetic system under consideration. In recent years, our ability to manipulate and measure single quantum systems have improved. It thus makes sense to speak about a fully quantum counterpart of the usually studied stimulus and response. We provide a quantum stimulus by placing a quantum state at one part of a complex system and study its quantum response: the quantum state produced at another part of the system after some time as a result of the placement. Quantum communication through a spin chain can be regarded as a specific example of the above study, where one puts a quantum state at one end of a specific complex system (namely the spin chain) and looks at how well the spin at the other end of the chain resembles that state as a function of time.

\section{THE SIMPLEST SPIN CHAIN QUANTUM COMMUNICATION PROTOCOL}

We will now present the simplest spin chain communication protocol that one can envisage and analyse its performance. The simplest possible spin chain is one composed of the lowest dimensional spin systems, namely spin-1/2 systems or qubits (so that the operators $S^{x}, S^{y}$ and $S^{z}$ can be replaced by the familiar Pauli matrices $\sigma^{x}, \sigma^{y}$ and $\left.\sigma^{z}\right)$. We also want to start our protocol with the spin chain initialized in a very simple state, such as one in which all spins are in the same pure state, say $|0\rangle$. We will have to choose the couplings $J_{i j}$ in Eq.(1) in such a manner that initialization of the spin chain to such a state is easy. Accordingly, we choose $J_{i j}<0$, which means that the spin chain describes a ferromagnet. The ground state of a ferromagnet in a magnetic field, however weak, is one where all spins point in the direction of the field. For example, all spins could be pointing down. In the simplest quantum communication protocol, Alice places an arbitrary quantum state at one 


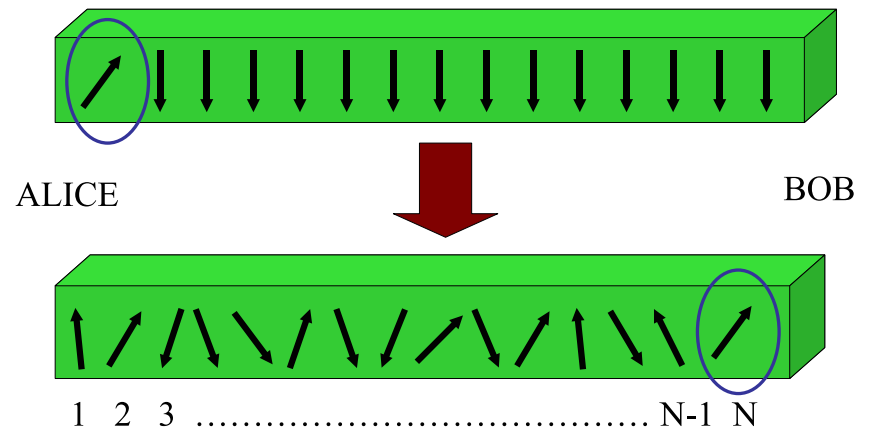

FIG. 5: The simplest spin chain communication protocol. A spin-1/2 ferromagnetic spin chain with all spins facing down is the quantum channel. Alice simply places a quantum state at one end of the chain and Bob simply picks up a close approximation of this state from his end of the spin chain after waiting a while.

end of the spin chain in such a "all down" state. This is depicted in the upper part of Fig [5 where Alice has placed an arbitrary state on the first spin of the chain, while all the other spins are still in the down state. Due to the natural evolution of the chain this state both disperses and propagates along the chain. As a result of this evolution, the state of the spin at Bob's end of the chain will vary with time. Bob now chooses an optimal moment of time in as long an interval that he can afford to wait to receive Alice's state. This moment of time is carefully chosen so that the state of the spin at Bob's end of the chain is as close as possible to the one that Alice intended to transmit. At this optimum time, Bob simply picks up the spin at his end of the chain, to conclude the communication protocol.

\section{A. Fidelity as a figure of merit}

In order to judge how well a quantum state is transferred by a spin chain one has to use a figure of merit. Suppose the state that Alice places into the spin at her end of the chain is depicted by $\left|\psi_{i n}\right\rangle$ and the state of the spin that Bob picks up at the optimum time $t_{0}$ is depicted in general by the density operator $\rho_{\text {out }}\left(t_{0}\right)$ (the output state is depicted by a density operator to allow for the possibility for it to be a mixed state). Then a measure of the quality of the transfer is defined by the fidelity

$$
F=\left\langle\psi_{\text {in }}\left|\rho_{\text {out }}\left(t_{0}\right)\right| \psi_{\text {in }}\right\rangle,
$$

which is always between 0 and 1 , with higher value meaning better transfer (it is unity for perfect transfer). A fidelity of $2 / 3$ can already be obtained if Alice simply measured her state, communicated the results classically to Bob and Bob simply reconstructed the state from this data. Thus $F$ needs to be greater than $2 / 3$ in our spin chain quantum communication scheme to be better than straightforward classical communication. Later, we will show that the use of another figure of merit, namely the amount of entanglement that can be transmitted by a spin chain, aids in the the justification that spin chains of arbitrary length are interesting as quantum communication channels.

\section{B. General formula for fidelity for arbitrary graphs}

I will first present the scheme in a general setting for arbitrary graphs of spins with ferromagnetic Heisenberg interactions and later proceed to the special cases motivated by realizability. Say there are $N$ spins in the graph and these are numbered $1,2, \ldots, N$. The Hamiltonian is given by

$$
\mathbf{H}_{\mathbf{G}}=-\sum_{<i, j>} J_{i j} \vec{\sigma}^{i} \cdot \vec{\sigma}^{j}-\sum_{i=1}^{N} B_{i} \sigma_{z}^{i} .
$$

$\vec{\sigma}^{i}=\left(\sigma_{x}^{i}, \sigma_{y}^{i}, \sigma_{z}^{i}\right)$ in which $\sigma_{x / y / z}^{i}$ are the Pauli matrices for the $i$ th spin, $B_{i}>0$ are static magnetic fields and $J_{i j}>0$ are coupling strengths, and $\langle i, j\rangle$ represents pairs of spins. $\mathbf{H}_{\mathbf{G}}$ describes an arbitrary ferromagnet with isotropic Heisenberg interactions. As mentioned above we initialize the graph in its ground state $|\mathbf{0}\rangle=|000 \ldots 0\rangle$ where $|0\rangle$ denotes the spin down state (i.e., spin aligned along $-z$ direction) of a spin. This can be accomplished easily for a ferromagnetic system by cooling. We will set the ground state energy $E_{0}=0$ (i.e., redefine $\mathbf{H}_{\mathbf{G}}$ as $E_{0}+\mathbf{H}_{\mathbf{G}}$ ) for the rest of this paper. We also introduce the class of states $|\mathbf{j}\rangle=|00 \ldots 010 \ldots . .0\rangle$ (where $\mathbf{j}=\mathbf{1}, \mathbf{2}, . . \mathbf{s}, . . \mathbf{r}, . ., \mathbf{N}$ ) in which the spin at the $j$ th site has been flipped to the $|1\rangle$ state. We now assume that the state sender Alice is located closest to the $s$ th (sender) spin and the state receiver Bob is located closest to the $r$ th (receiver) spin. All the other spins will be called channel spins. As mentioned before, to start the protocol, Alice simply places the state she wants to transmit to Bob on the $s$ th spin at time $t=0$. Let this state be $\left|\psi_{\text {in }}\right\rangle=\cos (\theta / 2)|0\rangle+e^{i \phi} \sin (\theta / 2)|1\rangle$. We can then describe the state of the whole chain at this instant (time $t=0$ ) as

$$
|\Psi(0)\rangle=\cos \frac{\theta}{2}|\mathbf{0}\rangle+e^{i \phi} \sin \frac{\theta}{2}|\mathbf{s}\rangle .
$$

Bob now waits for a specific time till the initial state $|\Psi(0)\rangle$ evolves to a final state which is as close as possible to $\cos \frac{\theta}{2}|\mathbf{0}\rangle+e^{i \phi} \sin \frac{\theta}{2}|\mathbf{r}\rangle$. As $\left[\mathbf{H}_{\mathbf{G}}, \sum_{i=1}^{N} \sigma_{z}^{i}\right]=0$, the state 
$|\mathbf{s}\rangle$ only evolves to states $|\mathbf{j}\rangle$ and the evolution of the spingraph (with $\hbar=1$ ) is

$$
|\Psi(t)\rangle=\cos \frac{\theta}{2}|\mathbf{0}\rangle+e^{i \phi} \sin \frac{\theta}{2} \sum_{\mathbf{j}=\mathbf{1}}^{\mathbf{N}}\left\langle\mathbf{j}\left|e^{-i \mathbf{H}_{\mathbf{G}} t}\right| \mathbf{s}\right\rangle|\mathbf{j}\rangle .
$$

The state of the $r$ th spin will, in general, be a mixed state, and can be obtained by tracing off the states of all other spins from $|\Psi(t)\rangle$. Undergraduate readers can familiarize themselves with this tracing procedure called partial tracing from quantum information textbooks 75], but essentially the density operator $\rho_{\text {out }}$ of the output state is obtained by $\operatorname{Tr}_{1,2, \ldots, N-1}(|\Psi(t)\rangle\langle\Psi(t)|)$, where $\operatorname{Tr}_{1,2, \ldots, N-1}$ means tracing over the states of the systems 1 to $N-1$. This evolves with time as

$$
\rho_{\text {out }}(t)=P(t)\left|\psi_{\text {out }}(t)\right\rangle\left\langle\psi_{\text {out }}(t)|+(1-P(t))| 0\right\rangle\langle 0|,
$$

with

$$
\left|\psi_{\text {out }}(t)\right\rangle=\frac{1}{\sqrt{P(t)}}\left(\cos \frac{\theta}{2}|0\rangle+e^{i \phi} \sin \frac{\theta}{2} f_{s, r}(t)|1\rangle\right),
$$

where $P(t)=\cos ^{2} \frac{\theta}{2}+\sin ^{2} \frac{\theta}{2}\left|f_{r, s}(t)\right|^{2}$ and $f_{r, s}(t)=$ $\left\langle\mathbf{r}\left|\exp \left\{-i \mathbf{H}_{\mathbf{G}} t\right\}\right| \mathbf{s}\right\rangle$. Note that $f_{r, s}(t)$ is just the transition amplitude of an excitation (the $|1\rangle$ state) from the $s$ th to the $r$ th site of a graph of $N$ spins.

Now suppose it is decided that Bob will pick up the $r$ th spin (and hence complete the communication protocol) at a predetermined time $t=t_{0}$. The fidelity of quantum communication through the channel averaged over all pure input states $\left|\psi_{i n}\right\rangle$ in the Bloch-sphere $\left((1 / 4 \pi) \int\left\langle\psi_{\text {in }}\left|\rho_{\text {out }}\left(t_{0}\right)\right| \psi_{\text {in }}\right\rangle d \Omega\right)$ is then

$$
F=\frac{\left|f_{r, s}\left(t_{0}\right)\right| \cos \gamma}{3}+\frac{\left|f_{r, s}\left(t_{0}\right)\right|^{2}}{6}+\frac{1}{2}
$$

where $\gamma=\arg \left\{f_{r, s}\left(t_{0}\right)\right\}$. To maximize the above average fidelity, we must choose the magnetic fields $B_{i}$ such that $\gamma$ is a multiple of $2 \pi$. Assuming this special choice of magnetic field value (which can always be made for any given $t_{0}$ ) to be a part of our protocol, we can simply replace $f_{r, s}\left(t_{0}\right)$ by $\left|f_{N, 1}\left(t_{0}\right)\right|$ in Eq.(8).

\section{Specifics for an open chain with nearest neighbour interactions}

As we intend to use a graph of interacting spins as a channel, we will use the most natural geometry for a channel, which is a linear open ended chain (Fig 5) with the sender (Alice) and the receiver (Bob) at opposite ends. To use an analytically solvable Hamiltonian $\mathbf{H}_{\mathbf{L}}$ we assume $J_{i j}=(J / 2) \delta_{i+1, j}$ (nearest neighbor interactions of equal strength) and $B_{i}=B$ (uniform magnetic field) for all $i$ and $j$ in Eq.(4) for $\mathbf{H}_{\mathbf{G}}$. The eigenstates of $\mathbf{H}_{\mathbf{L}}$, relevant to our problem are

$$
|\tilde{m}\rangle_{\mathbf{L}}=a_{m} \sum_{j=1}^{N} \cos \left\{\frac{\pi}{2 N}(m-1)(2 j-1)\right\}|\mathbf{j}\rangle,
$$

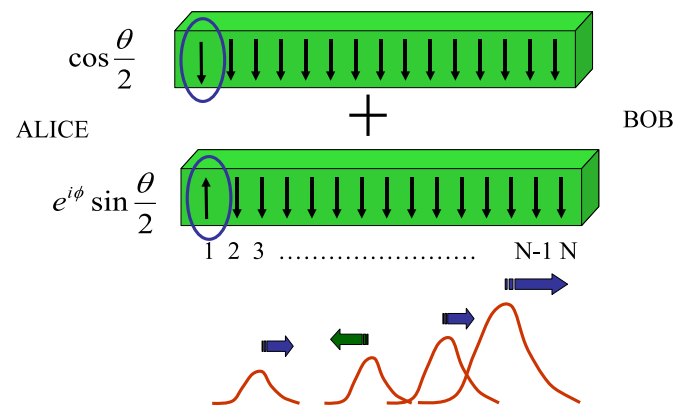

FIG. 6: The figure shows how quantum information is transmitted down a spin chain in the simplest protocol. The chain goes to a superposition of its ground state and a time evolving state. The time evolving state transmits a spin flip as a series of wave-packets which travels towards Bob while dispersing at the same time.

where $m=1,2, \ldots, N, a_{1}=1 / \sqrt{N}$ and $a_{m \neq 1}=\sqrt{2 / N}$ with energy (on setting $E_{0}=0$ ) given by $E_{m}=2 B+$ $2 J\left(1-\cos \left\{\frac{\pi}{N}(m-1)\right\}\right)$. In this case, $f_{r, s}\left(t_{0}\right)$ is given by

$$
f_{r, s}\left(t_{0}\right)=\sum_{m=1}^{N}\langle\mathbf{r} \mid \tilde{m}\rangle\langle\tilde{m} \mid \mathbf{s}\rangle e^{-i E_{m} t_{0}}=I D C T_{s}\left(v_{m, r}\right)
$$

where, $v_{m, r}=a_{m} \cos \left\{\frac{\pi}{2 N}(m-1)(2 r-1)\right\} e^{-i E_{m} t_{0}}$ and $I D C T_{s}\left(v_{m, r}\right)=\sum_{m=1}^{N} a_{m} v_{m, r} \cos \left\{\frac{\pi}{2 N}(m-1)(2 s-1)\right\}$ is the $s$ th element of the inverse discrete cosine transform of the vector $\left\{v_{m, r}\right\}$.

We now want to study the performance of our protocol for various chain lengths $N$ with $s=1$ and $r=N$ (Alice and Bob at opposite ends of the chain as shown in Fig(5). In general, Bob has to wait for different lengths of time $t_{0}$ for different chain lengths $N$, in order to obtain a high fidelity of quantum state transfer. The time $t_{0}$ for the highest fidelity in a given interval of time need not be related to $N$ by a simple formula. Why is this so? To understand the process, view Fig 6 . As shown in the figure, with an amplitude of $\cos \frac{\theta}{2}$ the spin chain remains in its ground state, while with an amplitude $e^{i \phi} \sin \frac{\theta}{2}$ the spin chain goes to a time evolving state in which the initial flip at Alice's end gets spread out (dispersed) and propagates as a series of waves, some propagating towards and some away from Bob. There is an initial high amplitude wavepacket which reaches Bob at a time of about $N / J$, but the highest peaks of $\left|f_{r, s}\right|$ at Bob's side are obtained by a constructive interference of several wave-packets. The specific time $t_{0}$ at which such a constructive interference takes place is not necessarily given by a closed form formula.

Using Eqs.(9) and (11), we can numerically evaluate 


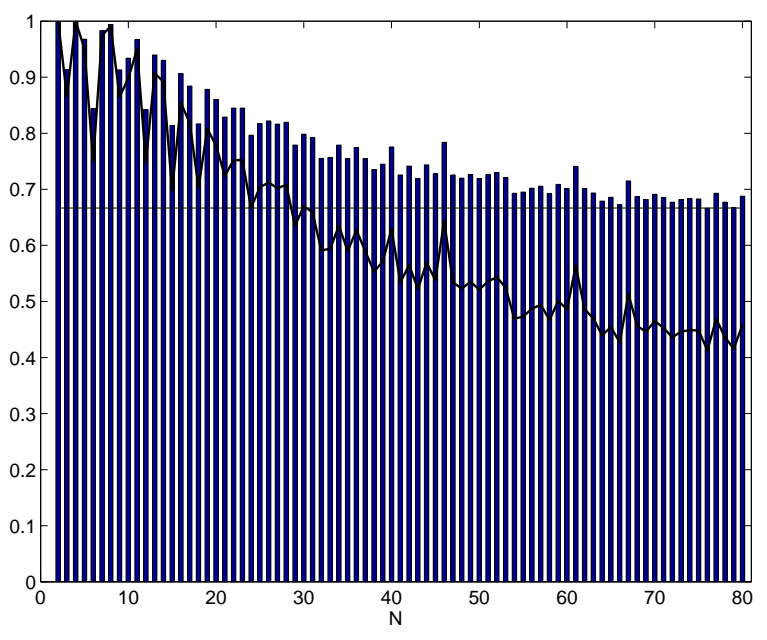

FIG. 7: The bar plot shows the maximum fidelity $F$ of quantum communication and the curve shows the maximum sharable entanglement $\mathcal{E}$ achieved in a time interval $[0,4000 / J]$ as a function of the chain length $N$ from 2 to 80. The time $t_{0}$ at which this maxima is achieved varies with $N$. The straight line at $F=2 / 3$ shows the highest fidelity for classical transmission of a quantum state.

the maximum of $\left|f_{N, 1}\left(t_{0}\right)\right|$ (which corresponds to the maxima of both fidelity and entanglement) for various chain lengths from $N=2$ to $N=80$ when Bob is allowed to choose $t_{0}$ within a finite (but long) time interval of length $T_{\max }=4000 / J$. This evaluation is fast because Eq.(11) allows us to use numerical packages for the discrete cosine transform. Taking a finite $T_{\max }$ is physically reasonable, as Bob cannot afford to wait indefinitely. It is to be understood that within $\left[0, T_{\max }\right]$, the time $t_{0}$ at which optimal quantum communication occurs varies with $N$. The maximum fidelities as a function of $N$ and the maximum amounts of entanglement sharable (both rounded to 3 decimal places) are shown in Fig 7 .

Fig 7 shows various interesting features of our protocol. The plot also shows that in addition to the trivial case of $N=2, N=4$ gives perfect $(F=1.000)$ quantum state transfer to 3 decimal places and $N=8$ gives near perfect $(F=0.994)$. The fidelity also exceeds 0.9 for $N=7,10,11,13$ and 14 . Till $N=21$ we observe that the fidelities are lower when $N$ is divisible by 3 in comparison to the fidelities for $N+1$ and $N+2$. While we do not have a clear cut explanation of this effect, it is obviously a link between number theory and constructive interference in a line. The plot also shows that a chain of $N$ as high as 80 exceeds the highest fidelity for classical transmission of the state i.e., $2 / 3$ in the time interval probed by us.

As an alternate system, one can also consider a ring of $2 N$ spins with Alice and Bob accessing the spins at diametrically opposite sites $(s=1, r=N+1)$. In that case, as discussed in Ref. [6] we find that the global maxima of $F$ coincides with that of the open chain of
$N$ qubits described above. This means that by using a ring one can communicate as efficiently over a distance $r-s=N$ as you can with a open ended line over a distance $r-s=N-1$. An immediate implication is that a 4 spin ring allows perfect communication between diametrically opposite sites (because a $N=2$ spin chain does so).

\section{Is an arbitrarily long spin chain a true quantum channel?-An answer through entanglement distribution}

With the results of the above section, it is not conclusive that arbitrarily long spin chains are truly "quantum" channels. Especially if even for a long interval $T_{\max }$, a 80 spin chain cannot transfer quantum states with a fidelity better than $2 / 3$, then can we regard a chain of say a 1000 spins as a quantum channel? It is true that if we increase $T_{\max }$, then longer and longer chains may yield a fidelity better than $2 / 3$ due to constructive interference at some time. But because the time is being obtained by an optimization over an arbitrarily large interval, this cannot be either proved or disproved easily.

We will thus consider a different figure of merit for judging the performance of the spin chain as a quantum communication channel, namely the amount of entanglement that can be transmitted through it. Entanglement is the truly "quantum" correlations that exist between two systems when they are in a inseparable state such as $\left|\psi^{+}\right\rangle=\frac{1}{\sqrt{2}}(|01\rangle+|10\rangle)$. The study of entanglement is a huge area of quantum information science, and we refer the reader to a review such as Ref. [87]. For our current purposes, it is sufficient to note that if Alice and Bob held one member each of a pair of particles in the state $\left|\psi^{+}\right\rangle$, then one of them could transmit a quantum state perfectly to the other using a celebrated protocol called quantum teleportation [88] (this transmission also requires some additional classical communication between Alice and Bob).

In particular, we will look at the transmission of the state of one member of a pair of particles in the entangled state $\left|\psi^{+}\right\rangle$through the spin chain channel. This is the usual procedure for sharing entanglement between separated parties through any channel. Alice prepares two qubits in the state $\left|\psi^{+}\right\rangle$, holds one of them (say, $A$ ) in her hand and places the other on site 1 of the chain. This procedure of putting in one member of an entangled state in a spin chain is illustrated in Fig, 8. After waiting for an optimal time $t_{0}$, Bob picks up the qubit $N$ from the chain. The joint state shared by Alice's qubit $A$ and Bob's qubit $N$ at this time is given by

$$
\begin{aligned}
\rho_{1 N}\left(t_{0}\right) & =\frac{1}{2}\left\{( 1 - | f _ { r , s } ( t _ { 0 } ) | ^ { 2 } ) | 0 0 \rangle \left\langle\left.00\right|_{1 N}\right.\right. \\
& \left.+\left(|10\rangle+\left|f_{r, s}\left(t_{0}\right)\right||01\rangle\right)\left(\left\langle 10|+| f_{r, s}\left(t_{0}\right)\right|\langle 01|\right)_{1 N}\right\}
\end{aligned}
$$

The entanglement $\mathcal{E}$ of the above state, as quantified by 


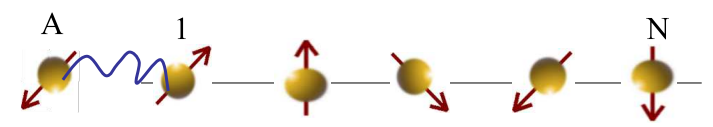

FIG. 8: The mechanism of transferring entanglement down a spin chain. The state of one member of a pair of qubits in a maximally entangled state is placed on the spin at Alice's end of the chain, while the other member is held by Alice. After a while, the spin at Bob's end of the chain will be entangled with the qubit held by Alice.

a certain measure called concurrence [89] is given by

$$
\mathcal{E}=\left|f_{r, s}\left(t_{0}\right)\right| .
$$

Thus, for any non-zero $f_{r, s}\left(t_{0}\right)$ (however small), some entanglement can be shared through the channel. This entanglement, being that of a $2 \times 2$ system, can also be distilled [90]. Distillation is a procedure whereby, if several copies of an entangled state such as $\rho_{1 N}\left(t_{0}\right)$ are shared in parallel between Alice and Bob, then it can be converted to a smaller collection of pure maximally entangled states $\left|\psi^{+}\right\rangle$shared between Alice and Bob. This procedure requires only local actions by Alice and Bob and classical communication between them. These $\left|\psi^{+}\right\rangle$ can subsequently be used for perfect transmission of a state from Alice to Bob by quantum teleportation.

We will now like to estimate the entanglement sharable through chains so large that the optimal $t_{0}$ identified by a numerical search in a long interval of time, is not good enough for an average fidelity higher than $2 / 3$. We will choose $t_{0}$ according to a fixed (in general, non-optimal) prescription and find how much entanglement can be transferred through the chain. To motivate this choice, we expand $e^{-i E_{m} t_{0}}$ in Eq.(11) as a Bessel function series to obtain

$$
\mathcal{E}=\left|\sum_{k=-\infty}^{\infty}(-1)^{N k}\left(J_{N+2 N k}\left(\beta_{0}\right)+i J_{N+2 N k}^{\prime}\left(\beta_{0}\right)\right)\right|,
$$

where $\beta_{0}=2 J t_{0}$. Using, $J_{N}\left(N+\xi N^{1 / 3}\right) \approx$ $(2 / N)^{1 / 3} A i\left(-2^{1 / 3} \xi\right)$ for large $N$ [118], (where $\operatorname{Ai}($.$) is$ the Airy function) we can prove that we get a maxima of $J_{N}\left(\beta_{0}\right)$ at $t_{0}=\left(N+0.8089 N^{1 / 3}\right) / 2 J$ and at this time

$$
\mathcal{E} \approx 2\left|J_{N}\left(\beta_{0}\right)\right| \approx 1.3499 N^{-1 / 3},
$$

which ranges from 0.135 for $N=1000$ to $1.35 \times 10^{-4}$ for $N=10^{12}$ (just 3 orders decrease in $\mathcal{E}$ for an increase in length $N$ by 9 orders - a very efficient way to distribute entanglement). Thus for any finite $N$, however large, the chain allows us to distribute entanglement of the order $N^{-1 / 3}$ in a time $t_{0}$ linear in $N$. An exchange coupled chain of any finite length is thus a bonafide quantum channel. It is worthwhile to point out that for an open ended $X Y$ spin chain (Hamiltonian $H^{X Y}$ ) the amount of entanglement $\mathcal{E}$ that can be transmitted by putting one member of an entangled pair of particles in the spin chain and waiting for a time $t_{0}=\left(N+0.8089 N^{1 / 3}\right) / 2 J$ is precisely twice the above amount (i.e., $2 \times 1.3499 N^{-1 / 3}$ ) 11].

\section{ROUTES TO PERFECTION AND NEAR PERFECTION}

The protocols of state transfer and entanglement distribution described above were primarily motivated by their simplicity. For the purposes of checking the quality of a qubit array and studying the quantum response to a quantum impulse - which are both important aims, these protocols should suffice. These transfer schemes, although imperfect, also already motivate the field of investigating quantum capacities of such spin chain channels [17, 48]. However, for connecting up two solid state quantum registers, which by far was our main motivation, it is only perfect or nearly perfect state transfer which is relevant. For example, to do a quantum gate between qubits in two separate registers, one needs to transfer the state of a qubit from the first register to the second perfectly, do a gate between that qubit and a qubit of the second register, and then transfer its state back to the first register perfectly. The protocols described above will not accomplish these tasks on their own. The second protocol (the one where one transmits the state of one member of a pair of entangled qubits through the channel) can be made useful when appended with entanglement distillation procedures. Basically, one has to first use the spin chain channel repeatedly to make Alice and Bob share several copies of the partially entangled state $\rho_{1 N}\left(t_{0}\right)$. Then entanglement distillation is performed to obtain a smaller number of pairs of particles in the state $\left|\psi^{+}\right\rangle$shared between Alice and Bob. The particles in the state $\left|\psi^{+}\right\rangle$can now be used to transmit a state perfectly, do quantum gates between qubits in well separated locations, and so forth. Clearly, it would be better if we could do perfect quantum state transfer through a spin chain without invoking an additional entanglement distillation process. With this view in mind, several schemes have been proposed which we present below.

\section{A. Engineered chains}

The couplings $J_{i j}$ can be carefully chosen (even when there are only nearest neighbor couplings, i.e., $i=j \pm 1$ ) to obtain a spin chain which accomplishes perfect quantum state transfer [9, 10, 16, 30]. We need to briefly digress to one of the very first systems studied in introductory quantum mechanics, namely a particle in a box, to clearly understand why this is possible. Let the box be a symmetric square well centered at $x=0$ (i.e., the potential satisfies $V(-x)=V(x))$ with infinitely high walls. 
Under these circumstances, the energy eigenstates $\phi_{k}(x)$ of $H$ with $k=0,1, \ldots \infty$ satisfy $\phi_{k}(-x)=(-1)^{k} \phi_{k}(x)$ (this property can be called alternating parity). The time evolution of any state $\psi(x)=\sum_{k} c_{k} \phi_{k}(x)$ is given by

$$
\psi(x, t)=\sum_{k} c_{k} e^{-i E_{k} t} \phi_{k}(x),
$$

where energies $E_{k} \propto k^{2}$ (such energies, which are proportional to integers, can be called commensurate energies). If one chooses a special time of evolution $t=\tau$ such that $E_{k} \tau=k^{2} \pi$, then we have

$$
\psi(x, \tau)=\sum_{k} c_{k}(-1)^{k} \phi_{k}(x)=\sum_{k} c_{k} \phi_{k}(-x)=\psi(-x) .
$$

Thus there is a time $\tau$ in which the complete wavefunction mirror inverts about $x=0$. This form of mirror inversion of a wave-function in an infinite square well have been known for some time in the quantum optics literature [91]. The properties which have been responsible for the mirror inversion detailed above are the alternating parity and the commensurate energy properties, and any other Hamiltonian which has the same features will also exhibit the above mirror inversion.

There is a close analogy between the position of a particle in a line and a single spin flip in a background of aligned spins in a spin chain. The position $x$ of the particle is analogous to the discretized positions $j=1, . ., N$ of the flip and the wavefunction $\psi(x)$ is simply analogous to a superposition $\sum_{j} c_{j}|\mathbf{j}\rangle$. If the couplings are (a) mirror symmetric about the centre of the spin chain (i.e., $J_{j, j+1}=J_{N-j, N-j+1}$ ), which gives the alternating parity property, and engineered to (b) give rise to commensurate energies, then at a certain time $\tau_{c}$ the state will mirror invert by the logic of the last paragraph, i.e., become $\sum_{j} c_{j}|\mathbf{N}-\mathbf{j}\rangle$. One form of engineering, which works for an open ended $X Y$ spin chain (Hamiltonian $\left.=\sum_{j} \mathbf{H}_{j, j+1}^{X Y}\right)$ with nearest neighbour interactions is $J_{j, j+1}=\sqrt{j(N-j)}$, which gives $E_{k} \propto k$ [9, 10]. The mirror inversion implies that in a time $\tau_{c}$ a spin flip at 1st site of the spin chain will be perfectly transferred to the $N$ th site i.e., $f_{1 N}\left(\tau_{c}\right)=1$, which in turn implies perfect quantum communication and entanglement transfer through formulae of Eqs.(91) and (12). Note that engineering a spin chain to obtain a commensurate spectrum can give different values of $J_{j, j+1}$ depending on the specific commensurate spectrum one chooses (such as one can choose $E_{k} \propto k, k^{2}, k(k+1)$ etc.) and engineered couplings other than that mentioned above have also been found [16, 33, 45]. In general, one chooses a specific commensurate spectrum and then finds the corresponding couplings by solving an inverse eigenvalue problem [20, 22]. Thus open ended XY spin chain with nearly uniform couplings [33], and non-nearest neighbour (dipolar) couplings [45], have been designed which can all accomplish perfect or near perfect quantum state transfer.

\section{B. Wave-packet encoding}

So far, we have been considering various protocols in which the $|1\rangle$ state to be transmitted by the chain is encoded on it as a single spin flip at one end. Remembering the analogy between positions $x$ of a particle in a line and discretized positions $j$ of a spin flip in a background of aligned spins, this is analogous to an infinitely narrow wavefunction (a Dirac delta function) of a particle in a line. For typical potentials such as a particle in a box or a harmonic oscillator, such a wavefunction is known to disperse (spread) rapidly in space and thus the particle's behaviour becomes very unlike that of a classical particle. However, it is possible to place particles in gaussian wave-packet states which have a low dispersion and travel with a definite group velocity, more or less as a classical particle would. Then any information encoded as a superposition of the presence and absence of a particle in such a state would travel more or less with a well defined velocity and reach the receiver at a predetermined time. Can one try a similar trick in the transmission of quantum information down a spin chain? Such a protocol was first proposed for a ring of $N$ spins interacting through the Heisenberg or the XY model [12], where "truncated" gaussian wavepacket states $\left|G\left(j_{0}, k\right)\right\rangle=\sum_{j} e^{-\left(j-j_{0}\right)^{2} / L^{2}} e^{-i k_{0} j}|\mathbf{j}\rangle$ centred at the site $j=j_{0}$ (and defined over $L$ sites around the site $j_{0}$ ) and with velocity $\propto k_{0}$ were used. In a block of $L$ spins near a site $j_{A}$, Alice encodes the $|1\rangle$ state of the qubit she wants to transmit on $\left|G\left(j_{A}, k\right)\right\rangle$ (the $|0\rangle$ is encoded as in the previous protocols). For appropriate choice of $k_{0}$ (see Ref.[12]) one can choose $L \sim N^{1 / 3}$, so that this wave-packet travels with a dispersion which is negligible and remains constant no matter how far it travels down the ring. Thus, Bob located at any distant site along the ring can catch almost the entire wavepacket by using a sufficiently long block of spins $\left(\sim N^{1 / 3}\right)$ to receive the state. To obtain the the fidelity of quantum communication and entanglement transfer using this wave-packet scheme, one simply has to replace $f_{1 N}$ in the earlier formulae Eqs.(9) and (12) by the amplitude of $\left|G\left(j_{A}, k\right)\right\rangle$ evolving to $\left|G\left(j_{B}, k\right)\right\rangle$ with time, where $j_{B}$ is the site around which Bob has access to a block of $\sim N^{1 / 3}$ spins. This amplitude can be as 95 percent for $N$ up to 5000 [12]. For open ended spin chains, it is not ideal for Alice to encode $|1\rangle$ in a truncated gaussian wavepacket at one end of the chain, as this will distort and spread when it reaches Bob at the other end of the chain. This problem has been cleverly resolved in Ref. [15], which shows that Alice can encode a different wave-form on a block of $N$ spins at one end, which evolves to a gaussian wave-packet of certain $k_{0}$ in the chain and travels to the other end with minimal dispersion. Additionally, Ref. [15] points out how such an encoding in blocks for near perfect communications is possible for any graph of spins (albeit, connected with each other through interactions) and how such an encoding/decoding can be accomplished by Alice/Bob with access only to a single spin 
each, but continuous time control of the interactions of these spins with the graph. Gaussian wave-packet encodings have also been suggested for communication through spin-chains in various static external fields [20, 52].

\section{Coupling qubits weakly to a quantum many body system}

Another approach is to couple the sending and receiving qubits weakly to a quantum many body system [20, 21, 40, 49]. Say the many body system is an arbitrary graph of spins which interact with each other with a coupling strength $J \sim 1$, while the sending and receiving qubits are coupled to the system through a coupling $\epsilon$ where $\epsilon<<1$. Moreover, assume all the couplings to be of $X Y$ or Heisenberg type (other interactions would also do as long as they can enable the transfer of an excitation through the graph from the sending to the receiving qubit). Then, one can derive effective $\mathrm{XY}$ or Heisenberg Hamiltonians between the two qubits when there are no eigenstates of the many-body system whose energy is close to 0 [20, 40, 49]. This is possible, for example, when the many-body system is in its ground state and has a finite energy gap $\Delta$ between the ground and the first excited state (such as a spin ladder [20]). Effectively, a Hamiltonian of the form $\epsilon^{2} \mathbf{H}_{s r}^{X Y}$ or $\epsilon^{2} \mathbf{H}_{s r}$ acts on them, where $s$ and $r$ stand for the sending and the receiving qubits respectively. The effective Hamiltonian can be rigorously derived using second order perturbation theory 20, 40, 49 . This case, which we will call the "off resonant" case [49], enables a perfect quantum state transfer by the simple fact that the two qubit Hamiltonians $\epsilon^{2} \mathbf{H}_{s r}^{X Y}$ or $\epsilon^{2} \mathbf{H}_{s r}$ do so in a time $t \sim 1 / \epsilon^{2}$. The other case is when the many-body system has exactly one available state $|\lambda\rangle$ of zero energy of the type in which a single spin is flipped from the ground state, and beyond that, there is a gap $\Delta$ to all other states of the single flip type. Then a "resonant" transfer [49] through the many body system takes place with an effective Hamiltonian $\epsilon \mathbf{H}_{s M}^{X Y}+\epsilon \mathbf{H}_{M r}^{X Y}$, where $\sigma_{M}^{x}, \sigma_{M}^{y}$ and $\sigma_{M}^{z}$ are defined for a delocalized qubit with $\sigma_{M}^{z}=+1$ corresponding to the many body system being in $|\lambda\rangle$ and $\sigma_{M}^{z}=-1$ corresponding to the many body system being in its ground state 21, 49]. This resonant effective Hamiltonian is just a three qubit $X Y$ spin chain which can perfectly transfer states in a time scale $t \sim 1 / \epsilon[9,22]$.

\section{Ising chain with global pulses}

Another approach is to use a spin chain Hamiltonian with a different type of coupling, namely a nearest neighbour Ising coupling, as given by

$$
\mathbf{H}^{I \text { sing }}=\sum_{j=1}^{N} J \sigma_{j}^{z} \sigma_{j+1}^{z},
$$

in conjunction with "global" pulses (pulses that act on each spin of the chain in exactly the same way) at regular intervals to perfectly transport a state from one of its ends to the other [36]. To understand this, we will need two unitary operations, one called the Hadamard (denoted by $H$ ) which acts on a single qubit to change $|0\rangle$ to $|+\rangle=|0\rangle+|1\rangle$ and $|1\rangle$ to $|-\rangle=|0\rangle-|1\rangle$ and the other called the Controlled- $Z$ or simply $C Z$, which acts on two qubits to change $|1\rangle| \pm\rangle$ to $|1\rangle|\mp\rangle$, but keep $|0\rangle| \pm\rangle$ unchanged [75]. It is shown in Ref.[36] that an Ising chain evolving on its own for a time $\pi / 4 J$ followed by fast (instantaneous) operations on the chain by global pulses at time $\pi / 4 J$ (and some extra operations, also fast, at the very ends of the spin chain), accomplishes the operation $S$ which is equivalent to a $C Z$ between all adjacent pairs of spins followed by a $H$ on each spin. The entire time evolution with interruptions by the instantaneous pulses at regular intervals is then equivalent to a series of applications of $S$. Now imagine a $N=4$ spin chain to be initialized in the state $\left(\alpha|0\rangle_{1}+\beta|1\rangle_{1}\right)|+\rangle_{2}|0\rangle_{3}|+\rangle_{4}$. Then successive applications of four $S$ operations accomplishes the evolution

$$
\begin{array}{cc} 
& \left(\alpha|0\rangle_{1}+\beta|1\rangle_{1}\right)|+\rangle_{2}|0\rangle_{3}|+\rangle_{4} \\
\stackrel{S}{\rightarrow} & \left(\alpha|+\rangle_{1}|0\rangle_{2}+\beta|-\rangle_{1}|1\rangle_{2}\right)|+\rangle_{3}|0\rangle_{4} \\
\stackrel{S}{\rightarrow} & |0\rangle_{1}\left(\alpha|+\rangle_{2}|0\rangle_{3}+\beta|-\rangle_{2}|1\rangle_{3}\right)|+\rangle_{4} \\
\stackrel{S}{\rightarrow} & |+\rangle_{1}|0\rangle_{2}\left(\alpha|+\rangle_{3}|0\rangle_{4}+\beta|-\rangle_{3}|1\rangle_{4}\right) \\
\stackrel{S}{\rightarrow} & |0\rangle_{1}|+\rangle_{2}|0\rangle_{3}\left(\alpha|+\rangle_{4}+\beta|-\rangle_{4}\right) .
\end{array}
$$

Thus an extra $H$ operation on each qubit (accomplishable by global pulses) after the above evolution has completely transferred the state at site 1 to site 4 . The authors of Ref.[36] show that in general, for a $N$ spin chain, one is able to transfer a quantum state from one end to the other by $N$ applications of $S$ (i.e. evolution of the chain till time $N \pi / 4 J$ interrupted by instantaneous pulses at regular intervals) and a $H$ on each qubit at the end of the evolution. Moreover, they also show that such a transfer can be accomplished by any starting state of the spin chain.

It should also be mentioned here that some very recent works show that if some degree of slow modulation of the couplings are allowed, then adiabatic passage can also be used to transfer quantum states perfectly through a spin chain channel [92, 93].

\section{PERFECTION WITH SIMPLICITY: A DUAL-RAIL PROTOCOL}

One always has to pay a "price" for perfect transfer. The original protocol with uniform couplings and single qubit encoding [6] necessitate mixed state entanglement distillation, and hence several uses of the channel even for the near perfect transmission of a single qubit. The en- 
gineering of couplings $[9,10,16,30]$ will be naturally restricted to those physical implementations where interaction strengths can be tuned to appropriate values, as opposed to being "given", while using wave-packets [12, 15] necessitate involving several qubits for encoding or continuous time control. Weak couplings [20, 21, 40, 49] may give a slower transfer, while global pulse schemes specialize to Ising chains [36]. We now present a protocol introduced in Ref. 18] which does not have to pay any of the above prices at the expense of using two spin chains in parallel as opposed to a single chain. The couplings in the spin chains need not be either uniform or specially engineered and could even be mildly random (with the reasonable assumptions that the chains are similar to each other and permit state transfer) 26] and the scheme involves only two qubits for encoding. We call this a dual rail protocol [18, 26].

An assumption of control at either end of the spin chain, of the spins which Alice and Bob control, is an implicit assumption in all communication protocols using spin chains. At the very least, Alice has to "swap in" a quantum state at one end of the chain (from her quantum register) which requires a tunable interaction of the first spin with a register spin. Bob has to have similar ability for retrieving the qubit from the other end of the chain. The same type of interaction, namely a switchable two qubit interaction, suffices for the encoding and decoding of this scheme involving parallel spin chains. We first describe below how parallel spin chains can be used for a "heralded" perfect quantum state transmission, where conditional on a positive outcome of a measurement, Bob can conclude that he has accurately received the state transmitted to him.

\section{A. A heralded perfect state transfer}

The idea of Ref. 18] is to use two spin chains $I$ and $I I$ in parallel as a single communication channel as shown in Fig9. As in previous protocols, this protocol is also restricted to a sector in which each spin chain has at most one spin flipped in a background of spins in the $|0\rangle$ state. We will thus use a similar notation as before for spin chain states, namely $|\mathbf{0}\rangle^{(I)}$ and $|\mathbf{0}\rangle^{(I I)}$ denoting the ferromagnetic ground states (all spins in the $|0\rangle$ state) of the chains $I$ and $I I$ respectively, and $|\mathbf{j}\rangle^{(I)}$ and $|\mathbf{j}\rangle^{(I I)}$ denoting the $j$ th spin flipped to the $|1\rangle$ state in the chains $I$ and II respectively. For the moment, we assume the chains to be non-interacting, identical copies of each other and coupled by uniform Heisenberg or $X Y$ interactions as in the original single chain based protocol [6] (some of these assumptions can be relaxed [26], as will be discussed later). The first spin of each chain is controlled by Alice, while the $N$ th spin of each chain is controlled by Bob. Initially, the spin chains are assumed to be in the state $|\mathbf{0}\rangle^{(I)}$ and $|\mathbf{0}\rangle^{(I I)}$. When Alice intends to transmit a qubit state $|\phi\rangle=\alpha|0\rangle+\beta|1\rangle$, she encodes this into the two spins that she controls as $|\tilde{\phi}\rangle=\alpha|01\rangle+\beta|10\rangle$. This encod-

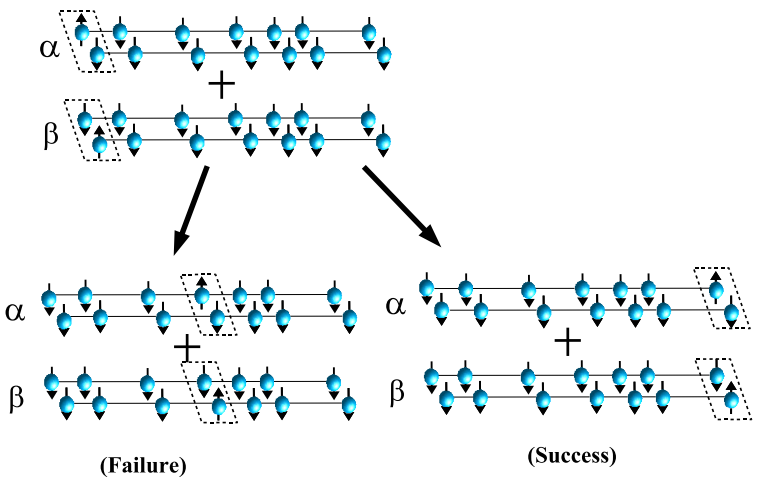

FIG. 9: The dual rail protocol for perfect quantum communications through spin chain channels. The upper part of the figure shows an arbitrary superposition of the logical qubit states being encoded at one end of the parallel chains (the dotted box shows the encoded states of a qubit). The lower parts of the figure show two possible states of the chain after the passage of some time and Bob's measurement. Bob's success corresponds to the state being received perfectly on the spins at his end of the chain. His failure corresponds to the information being retained in parts of the chain not accessed by Bob, as shown in the lower left hand side of the figure. Strictly speaking the state corresponding to failure will be a superposition of all states of the form shown in the lower left hand side i.e., it will be a superposition of all those states in which the dotted box is at sites other than $N$.

ing can be accomplished by a simple two qubit unitary operation (a two qubit quantum gate) [18] involving the qubits that Alice controls, and can be accomplished inside the quantum computer. This encoding places the entire system of two spin chains in the quantum state

$$
|\Phi(0)\rangle=\alpha|\mathbf{0}\rangle^{(I)}|\mathbf{1}\rangle^{(I I)}+\beta|\mathbf{1}\rangle^{(I)}|\mathbf{0}\rangle^{(I I)},
$$

which evolves with time as

$$
|\Phi(t)\rangle=\sum_{j=1}^{N} f_{1 j}(t)\left(\alpha|\mathbf{0}\rangle^{(I)}|\mathbf{j}\rangle^{(I I)}+\beta|\mathbf{j}\rangle^{(I)}|\mathbf{0}\rangle^{(I I)}\right) .
$$

It is then simple to calculate, by using the method of partial tracing as discussed before, the time varying density operator $\varrho(t)$ of the two spins which Bob controls, and is found to be

$$
\varrho(t)=\left(1-\left|f_{1 N}(t)\right|^{2}\right)|00\rangle\left\langle\left. 00|+| f_{1 N}(t)\right|^{2} \mid \tilde{\phi}\right\rangle\langle\tilde{\phi}| .
$$

Bob now measures the "total" spin component of his spins in the $z$ direction without measuring any of the spins individually. Such a measurement gives a value -1 for $|00\rangle$ and the value 0 for any superposition of $|01\rangle$ and $|10\rangle$. Physically, such a measurement can be accomplished by a coarse grained spin measurement on the two spins of Bob which is insensitive to the precise location of the magnetic moment (a parity measurement on the two spins [? ], which gives an outcome 0 for $|00\rangle$ and an outcome 1 for any superposition of $|01\rangle$ and $|10\rangle$ also suffices). When 
Bob gets the outcome -1 , which happens with probability $\left(1-\left|f_{1 N}(t)\right|^{2}\right)$, his spins are projected to the state $|00\rangle$ irrespective of the state transmitted. This heralds the failure on Bob's part to receive the state. On the other hand, when he obtains the outcome 0 , which happens with probability $\left|f_{1 N}(t)\right|^{2}$, his spins are projected to the state $|\tilde{\phi}\rangle$. Bob can now simply apply the inverse of the quantum gate that Alice used for encoding to his spins to obtain a decoded state $|\phi\rangle$, which corresponds to his perfect retrieval the state transmitted by Alice. Here we should point out that Bob's actions could also be combined into one two qubit quantum gate followed by a single qubit measurement, which the reader will find in the original presentation of this protocol [18]. For long Heisenberg and $X Y$ chains, thus, Bob's success probability in this heralded scheme scales as $\left|f_{1 N}(t)\right|^{2} \sim 1 / N^{2 / 3}$ in a time $t \sim O(N / J)$. In some sense, we have been able to convert the partial fidelity of the transmitted state in the original spin chain communication scheme to a probability of success, and when successful, Bob receives the transmitted state perfectly. It is easy to verify that all the above also holds for transmitting entanglement through the parallel spin chain channel. Thus if Alice and Bob were merely attempting to establish entanglement, then Alice could try to send the state of one member of an entangled pair of qubits through the channel. If Bob fails to receive the state, the channel is reset to the state $|\mathbf{0}\rangle^{(I)}|\mathbf{0}\rangle^{(I I)}$ (by cooling to the ground state in a magnetic field, for example) and Alice tries to transmit entanglement again. On average after attempting about $N^{2 / 3}$ times, each of which takes about $t \sim O(N / J)$ amount of time (so that the total time is $O\left(N^{1.67} / J\right)$ ), Alice and Bob will be able to share a pure maximally entangled state of two qubits such as $\left|\psi^{+}\right\rangle$.

\section{B. Unlimited enhancement of success probability by waiting}

Interestingly, if one was willing to wait till a time $O\left(N^{1.67} / J\right)$, then even the process of resetting the channel and repeatedly attempting to transmit the state (or entanglement, as described above) becomes unnecessary. When Bob fails, the state of the parallel spin chains is $\sum_{j=1}^{N-1} f_{1 j}(t)\left(\alpha|\mathbf{0}\rangle^{(I)}|\mathbf{j}\rangle^{(I I)}+\beta|\mathbf{j}\rangle^{(I)}|\mathbf{0}\rangle^{(I I)}\right)$. If we relabel $\sum_{j=1}^{N-1} f_{1 j}|\mathbf{j}\rangle^{(I)}$ and $\sum_{j=1}^{N-1} f_{1 j}|\mathbf{j}\rangle^{(I I)}$ as $|\varphi(t)\rangle^{(I)}$ and $|\varphi(t)\rangle^{(I I)}$, then this state can be rewritten as $\alpha|\mathbf{0}\rangle^{(I)}|\varphi(t)\rangle^{(I I)}+\beta|\varphi(t)\rangle^{(I)}|\mathbf{0}\rangle^{(I I)}$, which immediately clarifies to the reader that the initial quantum information is unspoilt and simply encoded in a delocalized form in the two chains. So can Bob try to retrieve the state again after waiting for a while? The state of the parallel chain system evolves, in another time $\tau$, to a state of the form $\sum_{j=1}^{N} \tilde{f_{1 j}}\left(\alpha|\mathbf{0}\rangle^{(I)}|\mathbf{j}\rangle^{(I I)}+\beta|\mathbf{j}\rangle^{(I)}|\mathbf{0}\rangle^{(I I)}\right)$, where $\tilde{f_{1 j}}$ can be simply expressed in terms of $f_{i j}(t)$ and $f_{i j}(\tau)$ [18]. This is just Eq.(20), with $f_{1 j}(t)$ replaced by $\tilde{f_{1 j}}$. Thus, Bob's actions (measurement and decoding) may again be repeated at a time $\tau$ after a failure, and again there is a probability of success equal to $\left|\tilde{f_{1 N}}\right|^{2}$. In this way, whenever Bob fails, he simply waits and again attempts to retrieve the state from the chain. In Ref.[18] it has been argued that the total probablity of success on repeated measurements by Bob can be made as high as 0.99 in a time scale of $\sim O\left(N^{1.67} / J\right)$.

Having read through the dual rail protocol, the reader may now ask some natural questions. For example, what happens if measurements of the same nature as Bob's were carried out at regular intervals even at other sites of the spin chain. Preliminary results indicate that the state transfer may then be significantly speeded up [32]. Another interesting question is whether there is any gain in going to multiple parallel chains as opposed to two? Indeed there is a gain in efficiency. While the dual rail protocol uses two spin chain channels to transmit a single qubit, multiple rails can be used to transmit a qubit per chain for a large number of rails if the states of multiple qubits are suitably encoded on Alice's end of the chain 24]. Recently, it has also been shown that even if the instantaneous measurements at fixed instants of time in the dual rail protocol were replaced by more realistic finite strength continuous measurements at the receiving end, the performance of the protocol can remain similar as long as of the strength of the measurement is appropriately tuned 94.

\section{Inherent robust aspects of the dual rail protocol}

The dual rail protocol is intrinsically robust in many ways. Suppose Bob has not measured his spins precisely at the optimal time, at which his probability of success is highest, but slightly before or after that time. The probability will still be quite high (as it is an analytic function of time), and when successful, Bob will still receive an unspoilt version of the state transmitted by Alice. This contrasts all previous protocols in which the fidelity itself of the transmitted state is affected by the time of its reception, and a non-optimal time results in the state being received by Bob being a somewhat corrupted version of the state transmitted by Alice. Another important robust aspect of the protocol stems from the fact the quantum state is transmitted through the parallel chains through what is called a "decoherence free" encoding. If the parallel chains are not that distant (this may be needed anyway to ensure the possibility of the quantum gates or joint measurements at the ends), any external environment couples to them only through their net magnetic moment in some given direction, such as through the operator $\sigma_{z}^{(I)}+\sigma_{z}^{(I I)}$. Alice encodes the state to be transmitted on her two spins as a superposition of states $|01\rangle$ and $|10\rangle$, which are eigenstates of $\sigma_{z}^{(I)}+\sigma_{z}^{(I I)}$ with eigenvalue 0 , and hence decoupled from the environment. The same holds during the transmission, as superpositions of states $|\mathbf{0}\rangle^{(I)}|\mathbf{j}\rangle^{(I I)}$ and $|\mathbf{j}\rangle^{(I)}|\mathbf{0}\rangle^{(I I)}$ are similarly decoupled from the environment. This decou- 
pling will enhance the time-scale over which the behavior of the parallel chain scheme is unaffected by an external environment relative to the time scale over which single spin chain based communication schemes remain unaffected. The above robustness aspects have been pointed out in more detail in Ref. [18], while Ref. [26] points out that the schemes are robust even to a mismatch of the chains with each other (the chains do not need to be identical copies of each other). In a nutshell, for mismatched rails which are not too dissimilar, there will be a series of times at which the absolute values of the amplitudes $f_{1 N}^{(I)}$ and $f_{1 N}^{(I I)}$ for the transmission of a flip from site 1 to $N$ through the chains $I$ and $I I$ will be coincident. If Bob performs his actions to receive the state at these specific times, then conditional on success, he will still receive an uncorrupted state [26].

\section{SINGLE CHAINS FOR PERFECT TRANSFER}

It is possible to obtain some protocols with similar positive attributes as the dual rail protocol which use only a single spin chain, which we discuss below.

\section{A. Chain of coupled qutrits}

Firstly, it is possible to use a single chain of higher dimensional quantum systems, such as qutrits (quantum three level systems) with levels $|+1\rangle,|0\rangle$ and $|-1\rangle$, instead of two parallel chains [18]. For our protocol, the qutrits should be coupled by the natural generalization of an exchange (or isotropic Heisenberg) interaction to higher dimensions given by a Hamiltonian $H=\sum_{i} P_{i, i+1}$ where

$$
P_{i, j}|\psi\rangle_{i}|\phi\rangle_{j}=|\phi\rangle_{i}|\psi\rangle_{j}
$$

One can look up Ref. 43] and references therein for a more detailed discussion of the above Hamiltonian. Please note carefully that in general, the above is not a Hamiltonian of a chain of coupled spin-1 systems (except for very special cases), but there are physical systems such as optical lattices, where it may be found [95]. For such a Hamiltonian, the state $|\mathbf{0}\rangle$ in which each qutrit is in the $|0\rangle$ state, is an eigenstate. From this state, one generates the states $|+\mathbf{j}\rangle$ and $|-\mathbf{j}\rangle$ of the chain in which the $j$ th qutrit is flipped to the $|+1\rangle$ and $|-1\rangle$ state respectively. Then the dual rail protocol described in the previous section can be exactly adapted to the chain of qutrits with the mappings $|\mathbf{0}\rangle^{(I)}|\mathbf{0}\rangle^{(I I)} \rightarrow|\mathbf{0}\rangle,|\mathbf{0}\rangle^{(I)}|\mathbf{j}\rangle^{(I I)} \rightarrow|+\mathbf{j}\rangle$ and $|\mathbf{j}\rangle^{(I)}|\mathbf{0}\rangle^{(I I)} \rightarrow|-\mathbf{j}\rangle$. One can check that Bob's measurement will now be mapped to a measurement which finds out whether his qutrit is in the state $|0\rangle$ or not (without ascertaining whether the qutrit is in the state $|+1\rangle$ or $|-1\rangle)$ and success is when he obtains the result "not $|0\rangle$ ". In a similar manner, if one had exchange coupled $d+1$ level systems, one could use one of those levels as the $|0\rangle$ state, and use the others to transmit a $d$ dimensional system perfectly with 0.99 probability of success in a time $\sim O\left(N^{1.67} / J\right)$ through the chain.

\section{B. Receiver with memory}

Curiously enough, even a single spin-1/2 chain without any encoding from Alice, can transmit a quantum state perfectly to Bob if he had a memory at his disposal [44]. As in all unencoded single chain protocols describe before, Alice simply places a state $\alpha|0\rangle+\beta|1\rangle$ on one end of a spin chain initialized in the state $|\mathbf{0}\rangle$. The clever trick used for receiving the state with arbitrarily high fidelity is to swap the state of Bob's qubit with that of a "fresh" memory qubit in state $|0\rangle$ at regular intervals [44]. The memory qubits are always non-interacting with each other and also non-interacting with the chain apart from during the swaps. Eventually, in a time scale which has been argued in Ref. [44] to be no larger than $O\left(N^{2} / J\right)$ for a broad class of chains (not necessarily uniform or Heisenberg or XY coupled), the spin chain ends up in the state $|\mathbf{0}\rangle$. In other words, all information about the input state is erased from the chain and transferred entirely to the collection of memory qubits. The unitarity of the whole evolution (the spin chain dynamics and the series of unitary swap operations) then guarantees that the collective state of all memory qubits is a function of $\alpha|0\rangle+\beta|1\rangle$. The same unitarity also guarantees that Bob can use another unitary operation to convert the state from a multiple qubit memory state to the single qubit state $\alpha|0\rangle+\beta|1\rangle$, thereby completing the reception of the state transmitted by Alice. Ref. [44] also shows that Alice can transmit many qubits simultaneously through the chain using the above protocol. Very recently there has been another interesting proposal in which Bob need possess only one memory qubit to receive a single qubit transmitted by Alice [59]. Bob lets this memory qubit interact with the spin at his end of the chain at regular intervals, but for different durations of time during each interaction. These times durations are so chosen that the entire amplitude of Bob's spin to be in the $|1\rangle$ state is transferred to the memory qubit (the reader may check Ref. 59. to satisfy him/herself that this is indeed possible through an unitary operation). In this way, as before, the chain will finally be left in the state $|\mathbf{0}\rangle$ (all information erased), and the memory qubit will end up in the state $\alpha|0\rangle+\beta|1\rangle$. One positive feature of this scheme is that the memory qubit may itself be a part of the spin chain, say an extra $N+1$ th spin attached to the $N$ th spin of the chain, with its interaction with the chain being switchable through a local magnetic field [59].

\section{PHYSICAL IMPLEMENTATIONS}

There has been several suggestions for the physical implementations of the quantum communication schemes 
described in this review. Essentially, chains of any physical system which has been proposed as a qubit, and which can be coupled with each other through an appropriate interaction (such as Heisenberg or XY), can be used. However, a permanent (non-tunable) coupling between the qubits will suffice. The most prominent class of suggestions are based on chains of superconducting qubits 25, 42, 51]. One such example is based on charge qubits [25], where the two states of the qubit are the presence or absence of a Cooper pair in a superconducting island. A Cooper pair can hop from one island to its neighbour through a Josephson coupling, which acts as an XY term in the Hamiltonian. There are additional parts to this Hamiltonian, such as a long range (much more than nearest neighbour) Coulomb interaction, which cannot be ignored [25]. Alternatively, one can use two opposite flux states of superconducting rings as the two states of a qubit, while these rings are coupled to each other through capacitive couplings [42, 51]. Implementations of slightly different schemes for entanglement distribution have also been discussed for Josephson junction arrays [100].

From the point of view of simulations with short chains, NMR is well suited (the ability to simulate communications through a 6 spin Heisenberg ring using benzene was already suggested in Ref.[6]). Recently, quantum communication through a 3 -qubit Ising chain using global pulses (the scheme of Ref. 36]) was demonstrated using NMR [101]. In quantum dots, which are tunnel coupled, so that electrons can freely hop from one dot to another, the electronic spin may be used as a qubit, and its transport in an array has been studied in a scheme slightly different from the ones discussed in this review [10]. Alternatively, excitons (coupled electron and hole pairs) in quantum dots may be used for implementing the schemes described in this review 96] (and other communication schemes as well [97]), with the two states of the qubit being the presence or absence of an exciton in a quantum dot, and an XY coupling between neighbouring dots being provided by the Förster interaction. There have also been various suggestions for implementations of the dynamics of XY chains in other systems, such as an array of low loss cavities for holding light coupled to each other so that photons can freely hop between them. When the light in each cavity is coupled to a single twolevel system, then the system simulates a XY model [98], and consequently, the schemes described here can also be implemented in such arrays. Another example is the simulation with chains of atoms trapped in optical lattices [99], which should again be an avenue for implementation.

\section{DEVELOPING AND FUTURE DIRECTIONS}

We would like to end the review by briefly pointing out the varied directions in which the topic is expanding as these offer the scope of much future work. One of the most obvious questions is what apart from quantum communications can be accomplished in the same spirit? By the "same spirit" we mean through the natural time evolution of a complex many body system. Starting from the most modest of aims, one can use a small ring of permanently coupled spins with a flux in the middle to design a quantum router [23]. In such a router the communication can be directed between any chosen pair of users from a multitude of users by adjusting the flux (an implicit assumption here is that it applies to those spin systems which involve charged entities at some level, so that a flux "twists" the boundary conditions of the ring). Routers have also been proposed in the context of weakly coupled sending/receiving qubit schemes [49]. Permanently coupled rings of spins can serve as quantum memories [102], and a time varying flux through such rings can undo the natural dispersion of quantum information stored in individual spins in such a system [103] (in general, spin rings with a flux is itself emerging as quite a fruitful system for varied quantum information applications [23, 103, 104, 105]). Networks of perpetually coupled spins can also serve as a quantum cloning machine, were information initially placed on $N$ of the spins is cloned to $M$ of the spins due to the natural dynamical evolution of the network [106, 107, 108]. Simple spin networks can also be automata for single spin measurement [53], while certain other desirable automata have been shown to be impossible [109]. What other dedicated small scale applications can we find for small (possibly engineered) networks of spins can thus be an interesting future goal.

Of course a more ambitious goal is to achieve fullscale quantum computation using permanently coupled systems such as spin chains. Indeed, it was noticed quite early (even predating the suggestion of spin chains for quantum communications) that the free evolution of small segments of $3-5$ Heisenberg/XY interacting spins can give rise to quantum gates between qubits encoded in these segments [11, 110], which was exploited for designing a universal quantum computer with spin chains [110]. Recently, natural evolutions of designer spin networks for quantum gates where qubits are fed in from one end of the system and read out from the other end of the system after gates have acted on them, have been proposed [105]. Interestingly, the free evolution of engineered spin chains can enact interesting multi-qubit quantum gates because of the exchange of fermionic operators to which such systems can be mapped [22, 111, 112]. Particularly, such spin chains can be used as a processor core for a quantum computer on to which states of qubits are loaded for running certain important classes of algorithms [112]. Whether one can find a general purpose processor core or whether a single permanently coupled network can be designed for running an entire quantum algorithm involving several gates, are interesting open questions.

Spin chains need not act merely as passive buses for quantum information, they can also act as sources of entanglement when put in an appropriate initial state. 
What we really want is a state which dynamically evolves in time and generates significant entanglement between the remotest parts of a spin chain. Probably the simplest example is flipping the spin at the middle of a ferromagnetic spin chain, and letting the state evolve, which can entangle the spins at the opposite ends of the chain [22]. Such studies have been conducted from very early on in the context of harmonic oscillator chains [13, 14] and also in context of graphs of qutrits [43] and oscillators [46], and more recently, also for graphs of spins [113] (see also Ref. [114] for entanglement from dynamics). However, this area, which one can call "quantum wires for entanglement generation and distribution", is open for future work as the possibilities of initial states of spin chains are enormous.

Another area offering possibilities for further exploration is when the spin chains, instead of being completely unmodulated, are subjected to a time varying external field. Pulsing the whole chain is an example, which is still a minimal procedure in comparison to switching individual interactions on and off. We have already encountered the fact that regular pulsing in an Ising chain can lead to perfect state transfer [36]. The same chain on appropriate pulsing can also accomplish universal quantum computation [36] (see also the work in Ref.[115]). For Heisenberg chains, pulsing with a field of an appropriate profile after flipping the spin at the middle of a ferromagnetic spin chain, gives rise to oppositely propagating entangled gaussian wave-packets, which would be very useful for the distribution of entanglement [56]. Applying external fields to dimerized spin chains can also enable encoding qubits in domain walls and by varying the profile of this field, such qubits can be transported [116]. It remains an open question as to what else can be accomplished by time varying external fields on spin chains. This question is particularly interesting because modulating a few external parameters can control how correlations propagate in spin chains [119], and the implications of that for quantum communications is worthwhile to examine.

Another open area is related to the issue of quantum response of a physical system to a quantum impulse as mentioned in the introduction. Quantum communication through a spin chain is one example of this, with the fidelity of transmission being a kind of response function. In this context, Ref. 62 finds, for the scenario where Alice's and Bob's qubits are weakly coupled to a spin system, that quantum phase transitions of the spin system can be detected by a drop in this response function. When Alice's and Bob's spins are coupled as strongly to the spin system as the spins in the system are coupled to each other, then Ref. 63] reports the opposite behaviour, namely that the same response function peaks at some quantum phase transitions. Ref. [63] is also an example of proceeding to higher spins in the context of quantum communication using spin chains. This is also an interesting direction. It has been shown, for example, that chains of higher dimensional systems coupled through the permutation Hamiltonian of subsection $\mathrm{VA}$ can be used to distribute much more entanglement than possible through a spin-1/2 chain [122]. Another example is a lattice system in which a large number of bosons can sit at each site, which has been shown to be able to distribute more and more entanglement as the number of bosons is increased [121]. Whether generically chains of higher spin systems provides a better quantum communication bus in comparison to low spin systems is an interesting open question.

Some directions of investigation are important for realistic physical implementations. For example, what happens when spins interact through long range dipolar interactions? Then it has been found that state transfer process in an uniformly coupled chain reaches near unit fidelity at the expense of a time which scales as $N^{3} / J$ [57]. Effect of randomness [50, 58] and defects [123] on the quality of quantum information transmission has also been studied, as well thermal effects [28]. Of course, a question of central importance is what happens if the spins of the chain are not isolated, but coupled to their environment, as such a coupling might be unavoidable in certain physical implementations. When each spin of an exchange coupled system is coupled to its own independent bath of polarized spins through a XY coupling, then, it has been shown that the fidelity of communication is unaffected 61]. Only the time of communication is delayed and the fidelity undergoes rapid oscillations with time [61]. Effects of other kinds of baths on spin chain quantum communications have also been recently analyzed [55, 120]. No doubt the investigations of the above kinds of issues which will automatically arise in the context of practical implementations, will form a major part of future research on quantum communications through spin chain dynamics.

\section{ACKNOWLEDGEMENTS}

I would like to particularly thank Daniel Burgarth, the chief architect of the dual rail protocol, and my collaborator in many of the papers covered here. I would also like to thank my other collaborators in various papers mentioned in this review, namely, Vittorio Giovannetti, Vladimir Korepin, Vlatko Vedral, Man-Hong Yung, Simon Benjamin, Debbie Leung, Christopher Hadley, Alessio Serafini, Dimitris Angelakis, Kurt Jacobs, Kosuke Shizume, Bai-Qi Jin, Yasser Omar, Antonio Costa, Jens Eisert, Martin Plenio, Benoit Vaucher, Tania Montiero, Tom Boness, Andrew Fisher and Martina Avellino. I thank EPSRC for an Advanced Research Fellowship and for support through the grant GR/S62796/01 and the QIPIRC (GR/S82176/01). 
[1] C.H. Bennett and G. Brassard, "Quantum Cryptography: Public Key Distribution and Coin Tossing", Proceedings of IEEE International Conference on Computers Systems and Signal Processing, Bangalore India, December 1984, pp 175-179.

[2] A. K. Ekert, Phys. Rev. Lett. 67, 661 (1991).

[3] D. Kielpinski, C. Monroe and D. J. Wineland, Nature 417, 709 (2002).

[4] B. B. Blinov, D. L. Moehring, L.-M. Duan, C. Monroe, Nature 428, 153 (2004).

[5] A. J. Skinner, M. E. Davenport, and B. E. Kane, Phys. Rev. Lett. 90, 087901 (2003).

[6] S. Bose, Phys. Rev. Lett 91, 207901(2003).

[7] V. Subrahmanyam, Phys. Rev. A 69, 034304 (2004).

[8] L. Amico, A. Osterloh, F. Plastina, R. Fazio and G. M. Palma, Phys. Rev. A. 69, 022304 (2004).

[9] M. Christandl, N. Datta, A. Ekert and A. J. Landahl, Phys. Rev. Lett. 92, 187902 (2004).

[10] G. M. Nikolopoulos, D.Petrosyan and P. Lambropoulos, Europhys. Lett. 65, 297 (2004).

[11] M.-H. Yung, D. W. Leung and S. Bose, Quan. Inf. Com. 4, 174 (2003).

[12] T. J. Osborne and N. Linden, Phys. Rev. A 69, 052315 (2004).

[13] J. Eisert, M.B. Plenio, S. Bose, J. Hartley, Phys. Rev. Lett. 93, 190402 (2004).

[14] M.B. Plenio, J. Hartley and J. Eisert, New J. Phys. 6, 36 (2004).

[15] H. L. Haselgrove, Phys. Rev. A 72, 062326 (2005).

[16] C. Albanese, M. Christandl, N. Datta and A. Ekert, Phys. Rev. Lett. 93, 230502 (2004).

[17] V. Giovannetti and R. Fazio, Phys. Rev. A 71, 032314 (2005).

[18] D. Burgarth and S. Bose, Phys. Rev. A 71, 052315 (2005).

[19] Y.Li, T.Shi, B.Chen, Z.Song and C.P.Sun, Phys. Rev. A 71, 022301 (2005).

[20] T. Shi, Ying Li, Z. Song and C. P. Sun, Phys. Rev. A 71, 032309 (2005).

[21] M. B. Plenio and F. L. Semiao, New J. Phys. 7, 73 (2005).

[22] M.-H. Yung and S. Bose, Phys. Rev. A 71, 032310 (2005).

[23] S. Bose, B.-Q. Jin, and V. E. Korepin, Phys. Rev. A 72, 022345 (2005).

[24] D. Burgarth, V. Giovannetti and S. Bose, J. Phys. A: Math. Gen. 38, 6793 (2005).

[25] A. Romito, R. Fazio and C. Bruder, Phys. Rev. B 71, 100501(R) (2005).

[26] D. Burgarth and S. Bose, New J. Phys. 7, 135 (2005).

[27] M. Paternostro, G. M. Palma, M. S. Kim, G. Falci, Phys. Rev. A 71, 042311 (2005).

[28] A. Bayat and V. Karimipour Phys. Rev. A 71, 042330 (2005).

[29] Y. Li, Z. Song, C.P. Sun, quant-ph/0504175

[30] M. Christandl, N. Datta, T. C. Dorlas, A. Ekert, A. Kay, and A. J. Landahl, Phys. Rev. A 71, 032312 (2005).

[31] G. De Chiara, D. Rossini, S. Montangero and R. Fazio, Phys. Rev. A 72, 012323 (2005).

[32] B. Vaucher, D. Burgarth and S. Bose, J. Opt. B: Quantum Semiclass. Opt. 7, S356-S362 (2005).
[33] P. Karbach and J. Stolze, Phys. Rev. A 72, 030301 (2005).

[34] S. Yang, Z. Song and C. P. Sun, Phys. Rev. A 73, 022317 (2006).

[35] J. Fitzsimons and J. Twamley, Phys. Rev. A 72, 050301(R) (2005).

[36] J. Fitzsimons and J. Twamley, Phys. Rev. Lett. 97, 090502 (2006).

[37] Andrew D. Greentree, Simon J. Devitt and Lloyd C. L. Hollenberg, Phys. Rev. A 73, 032319 (2006).

[38] Xiao-Feng Qian, Ying Li, Yong Li, Z. Song and C. P. Sun, Phys. Rev. A 72, 062329 (2005).

[39] S. D. Hamieh and M. I. Katsnelson, Phys. Rev. A 72, $032316(2005)$.

[40] A. Wojcik, T. Luczak, P. Kurzyski, A. Grudka, T. Gdala and M. Bednarska, Phys. Rev. A 72, 034303 (2005).

[41] M. Paternostro, M. S. Kim, E. Park and J. Lee, Phys. Rev. A 72, 052307 (2005).

[42] A. O. Lyakhov and C. Bruder, New J. Phys. 7, 181 (2005).

[43] C. Hadley, A. Serafini and S. Bose, Phys. Rev. A 72, 052333 (2005).

[44] V. Giovannetti and D. Burgarth, Phys. Rev. Lett. 96, 030501 (2006).

[45] A. Kay, Phys. Rev. A 73, 032306 (2006).

[46] A. Perales and M. B. Plenio, J. Opt. B: Quantum Semiclass. Opt. 7, S601-S609 (2005).

[47] S. Yang, Z. Song and C.P. Sun, Phys. Rev. A 73, 022317 (2006).

[48] D. Rossini, V. Giovannetti and R. Fazio, Int. J. Quantum Info. (to appear), quant-ph/0609022

[49] A. Wojcik, T. Luczak, P. Kurzynski, A. Grudka, T. Gdala, M. Bednarska, Phys. Rev. A 75, 022330 (2007).

[50] G. De Chiara, D. Rossini, S. Montangero and R. Fazio, Phys. Rev. A 72, 012323 (2005).

[51] A. Lyakhov and C. Bruder, Phys. Rev. B 74, 235303 (2006).

[52] B. Chen and Z. Song, Comm. Th. Phys. 46, 749 (2006).

[53] A. Kay, Phys. Rev. Lett. 98, 010501 (2007).

[54] F. de Pasquale, G. Giorgi and S. Paganelli, Phys. Rev. A 74, 012316 (2006).

[55] J.-M. Cai, Z.-W. Zhou and G.-C. Guo, Phys. Rev. A 74, 022328 (2006).

[56] T. Boness, S. Bose and T. S. Monteiro, Phys. Rev. Lett. 96, 187201 (2006).

[57] M. Avellino, A.J. Fisher and S. Bose, Phys. Rev. A 74, 012321 (2006).

[58] J.P. Keating, N. Linden, J.C.F. Matthews and A. Winter, quant-ph/0606205

[59] D. Burgarth, V. Giovannetti and S. Bose, quant-ph/0610018

[60] M.-H. Yung, Phys. Rev. A 74, 030303 (2006).

[61] D. Burgarth and S. Bose, Phys. Rev. A 73, 062321 (2006).

[62] Michael J. Hartmann, Moritz E. Reuter and Martin B. Plenio, New J. Phys. 8, 94 (2006).

[63] O. Romero-Isart, K. Eckert and A. Sanpera, quant-ph/0610210

[64] M. Arnesen, S. Bose, and V. Vedral, Phys. Rev. Lett. 87, 017901 (2000).

[65] A. Osterloh, L. Amico, G. Falci, and R. Fazio, Nature 
416, 608 (2002).

[66] T. Osborne and M. Nielsen, Phys. Rev. A 66, 032110 (2002).

[67] G. Vidal, J. Latorre, E. Rico, and A. Kitaev, Phys.Rev. Lett. 90, 227902 (2003).

[68] A. Osterloh, L. Amico, R. Fazio and V. Vedral, quant-ph/0703044.

[69] F. Verstraete, M.A. Martin-Delgado, J.I. Cirac, Phys. Rev. Lett. 92, 087201 (2004).

[70] L. Campos Venuti, C. Degli Esposti Boschi, M. Roncaglia, Phys. Rev. Lett. 96, 247206 (2006).

[71] J. A. Hoyos and G. Rigolin, Phys. Rev. A 74, 062324 (2006).

[72] D. Deutsch and R. Jozsa, Proc. R. Soc. Lond. A 439, 553 (1992).

[73] P. Shor, Proc. 35th Ann. Symp. on Found. of Computer Science (IEEE Comp. Soc. Press, Los Alamitos, CA, 1994) 124-134.

[74] L.K. Grover, Phys. Rev. Lett. 79, 4709 (1997).

[75] M. A. Nielsen and I. L. Chuang (2000). Quantum computation and quantum information. Cambridge University Press.

[76] R. Feynman, Intl J. of Theo. Phys. 21, 467 (1982).

[77] J. I. Cirac and P. Zoller, Phys. Rev. Lett. 74, 4091 (1995).

[78] A. Imamoglu, D. D. Awschalom, G. Burkard, D. P. DiVincenzo, D. Loss, M. Sherwin and A. Small, Phys. Rev. Lett. 83, 4204 (1999).

[79] M. R. Geller and A. N. Cleland, Phys. Rev. A 71, 032311 (2005).

[80] D. Loss and D. P. DiVincenzo, Phys. Rev. A 57, 120 (1998).

[81] B. E. Kane, Nature 393, 133 (1998).

[82] C. H. W. Barnes, J. M. Shilton and A. M. Robinson, Phys. Rev. B 62, 8410 (2000).

[83] R. Vrijen and E. Yablonovitch, Physica E 10, 569 (2001).

[84] A. T. Costa Jr, S. Bose and Y. Omar, Phys. Rev. Lett. 87, 277901 (2001).

[85] M. Oskin, F. T. Chong, I. L. Chuang and J. Kubiatowicz, Proceedings of the 30th International Symposium on Computer Architecture (ISCA 2003).

[86] P. R. Hammar et. al., Phys. Rev. B 59, 1008 (1999).

[87] M. B. Plenio and V. Vedral, Contemp. Phys. 39, 431466 (1998).

[88] C. H. Bennett et. al., Phys. Rev. Lett. 70, 1895 (1993).

[89] W. K. Wootters, Phys. Rev. Lett. 80, 2245-2248 (1998).

[90] M. Horodecki, P. Horodecki, and R. Horodecki, Phys. Rev. Lett. 78, 574-577 (1997).

[91] David L. Aronstein and C. R. Stroud, Jr. Phys. Rev. A 55, 4526 (1997).

[92] T. Ohshima, A. Ekert, D. K. L. Oi, D. Kaslizowski and L. C. Kwek, quant-ph/0702019.

[93] K. Eckert, O. Romero-Isart and A. Sanpera, quant-ph/0702082
[94] K. Shizume, K. Jacobs, D. Burgarth and S. Bose, quant-ph/0702029.

[95] C. Hadley and S. Bose, quant-ph/0602139

[96] Irene D'Amico, Microelectronics Journal 37, 1440 (2006).

[97] Timothy P. Spiller, Irene D'Amico and Brendon W. Lovett, New J. Phys. 9, 20 (2007).

[98] D. G. Angelakis, M. F. Santos and S. Bose, quant-ph/0606159

[99] L.-M. Duan, E. Demler and M. D. Lukin, Phys. Rev. Lett. 91, 090402 (2003).

[100] D.I. Tsomokos, M.J. Hartmann, S.F. Huelga and M.B. Plenio, New. J. Phys. (to appear), quant-ph/0611077.

[101] J. Fitzsimons, L. Xiao, S. C. Benjamin and J. A. Jones, quant-ph/0606188.

[102] Z. Song and C. P. Sun, Fizika Nizkikh Temperatur 31, $686(2005)$.

[103] S. M. Giampaolo, F. Illuminati, A. Di Lisi and S. De Siena, Laser Physics 16, 1411 (2006).

[104] K. Maruyama, T. Iitaka and F. Nori, Phys. Rev. A 75, 012325 (2007).

[105] A. Kay and M. Ericsson, New J. Phys. 7, 143 (2005).

[106] G. De Chiara, R. Fazio, C. Macchiavello, S. Montangero and G. M. Palma, Phys. Rev. A 70, 062308 (2004).

[107] G. De Chiara, R. Fazio, C. Macchiavello, S. Montangero and G. M. Palma, Phys. Rev. A 72, 012328 (2005).

[108] Q. Chen, J. Cheng, K.-L. Wang and J. Du, Phys. Rev. A 74, 034303 (2006).

[109] A. Kay, quant-ph/0702088

[110] S. C. Benjamin and S. Bose, Phys. Rev. Lett. 90, 247901 (2003).

[111] S. R. Clark, C. Moura Alves and D. Jaksch, New J. Phys. 7, 124 (2005).

[112] Man-Hong Yung, Simon C. Benjamin and Sougato Bose, Phys. Rev. Lett. 96, 220501 (2006).

[113] I. D'Amico, B. W. Lovett and T. P. Spiller, quant-ph/0702269

[114] Aditi Sen De, Ujjwal Sen, Maciej Lewenstein, Phys. Rev. A 72, 052319 (2005).

[115] R. Raussendorf, Phys. Rev. A 72, 052301 (2005).

[116] Vanita Srinivasa, Jeremy Levy and C. Stephen Hellberg, quant-ph/0606089.

[117] M. Koniorczyk, P. Rapan and V. Buzek, Phys. Rev. A 72, 022321 (2005).

[118] M. Abramowitz and I. A. Stegun, Handbook of Mathematical Functions (Dover, New York, 1972).

[119] T. S. Cubitt and J.I. Cirac, quant-ph/0701053

[120] Lan Zhou, Jing Lu, Tao Shi, C. P. Sun, quant-ph/0608135.

[121] S. Bose, cond-mat/0610024

[122] A. Bayat and V. Karimipour, Phys. Rev. A 75, 022321 (2007).

[123] Tony J. G. Apollaro, Francesco Plastina, Phys. Rev. A 74, 062316 (2006). 